\title{
Can Water Polarizability Be Ignored in Hydrogen Bond Kinetics?
}

\author{
Huafeng Xu, Harry A. Stern, and B. J. Berne* \\ Department of Chemistry and Center for Biomolecular Simulation, Columbia University, \\ 3000 Broadway, New York, New York 10027
}

Received: September 7, 2001; In Final Form: December 6, 2001

\begin{abstract}
The kinetics of forming and breaking water-water hydrogen bonds in neat water, an aqueous solution of ethane, and an aqueous solution of $\mathrm{NaCl}$ are studied by molecular dynamics simulations. We compare nonpolarizable and polarizable water models to elucidate the effect of water's polarizability on hydrogen bonds. We find that polarizability strengthens the hydrogen bonds and increases the hydrogen bond relaxation time by a factor of between $50 \%$ and $100 \%$. The Gibbs energy of activation for breaking hydrogen bonds is $\sim 0.2 \mathrm{kcal} \cdot \mathrm{mol}^{-1}$ higher for the polarizable water model. Polarizability also causes the rate of forming and breaking hydrogen bonds to be more dependent on the local environment.
\end{abstract}

\section{Introduction}

The unique properties of water depend fundamentally on its ability to form hydrogen bonds. ${ }^{1,2}$ According to quantum chemistry, hydrogen bonding in small water clusters is cooperative in nature. The hydrogen bond in water dimers is weaker than in water trimers and tetramers. This cannot be explained by nonpolarizable force fields; thus, it might be expected that realistic modeling of hydrogen bond strengths and dynamics in neat water and in aqueous solutions would require the use of polarizable force fields.

The elementary process of forming and breaking water-water hydrogen bonds plays a crucial role in the dynamic behavior of liquid water. ${ }^{3}$ Consequently, water-water hydrogen bond kinetics has been the subject of intensive study. ${ }^{4-9}$ Molecular dynamics simulations, which are able to provide atomistic resolution of the dynamic process, have contributed greatly to the understanding of water-water hydrogen bond kinetics. ${ }^{7,9-14}$ Because the preponderance of these molecular dynamics simulations are based on nonpolarizable force fields, it is important to establish whether these simpler models are capable of providing an accurate treatment of hydrogen bond kinetics. With the introduction of new polarizable force fields such as the TIP4P/FQ force field and with new methodologies for speeding up molecular dynamics such as P3ME/r-RESPA, ${ }^{15}$ it is now possible to address this question. In this article we present results for neat water, and dilute aqueous solutions of $\mathrm{NaCl}$ and ethane. These studies show that polarizable water models lead to a different picture of hydrogen bond kinetics, one that is more in accord with experiment than nonpolarizable models. These observations led us to suggest that whenever possible polarizable force fields should be used for modeling dynamic processes in aqueous solutions. These new force fields are easily incorporated into molecular dynamics, and their use does not cost much more than the use of nonpolarizable force fields.

Nonpolarizable water models are unable to describe the response of the electronic structure to a varying environment. Experimentally, water has a nonzero and nearly isotropic polarizability: $\left(\alpha_{x x}, \alpha_{y y}, \alpha_{z z}\right)=\left(1.47 \AA^{3}, 1.53 \AA^{3}, 1.42 \AA^{3}\right) .{ }^{16}$ General dynamic processes, such as translational and rotational

* Corresponding author. E-mail: berne@chem.columbia.edu. diffusion, as well as dielectric relaxation, tend to be slower in polarizable water models, and more in agreement with experimental values, than in fixed-charge water models. ${ }^{17}$ The coordination number of hydrated anions is sensitive to polarizability. In nonpolarizable water the coordination number of the $\mathrm{Cl}^{-}$ion is approximately seven, whereas in polarizable water it is six. ${ }^{18,19}$ Polarizable models also predict fine details such as translational rattling effects observed in dielectric relaxation experiments, which are absent in nonpolarizable models. ${ }^{17}$

The presence of polarizability gives rise to many-body interactions, as a consequence of which the water-water interaction will not only depend on the pair's mutual position and orientation but also on its environment. The electric field from nearest neighbors induces changes in the electronic configuration of the water molecules, which in turn affect their interaction with other water molecules. This will give rise to cooperativity in the behavior of water. In particular, when a water molecule forms a hydrogen bond, it undergoes an internal rearrangement of charge density, which generally strengthens other hydrogen bonds formed by the same water. Therefore, we expect to see mutual enhancement of hydrogen bond strength because of water polarizability. This should produce cooperative behavior in hydrogen bond kinetics, which has not been observed in other studies with nonpolarizable water models. ${ }^{11}$ We explore such cooperativity in this article.

\section{Simulation Methods}

We performed molecular dynamics simulations for neat water, an aqueous solution of ethane, and an aqueous solution of $\mathrm{NaCl}$, with use of both polarizable and nonpolarizable water models. For simulations with nonpolarizable water we used the TIP4P model of Jorgensen et al. ${ }^{20}$ and the SPC/E model of Berendsen et al. ${ }^{21}$ For simulations with polarizable water we used the TIP4P/FQ model of Rick et al. ${ }^{17}$ The TIP4P/FQ model shares the same geometry as the TIP4P model but it allows the partial charges on the molecule to fluctuate dynamically in response to changes in the electrostatic environment. As in the original article, we treated the fluctuating charges as dynamic variables and used the extended Lagrangian method ${ }^{17,22-24}$ to propagate them. The OPLS/AA force field ${ }^{25}$ (a nonpolarizable force field) was used for the solute molecules. Standard OPLS combining 


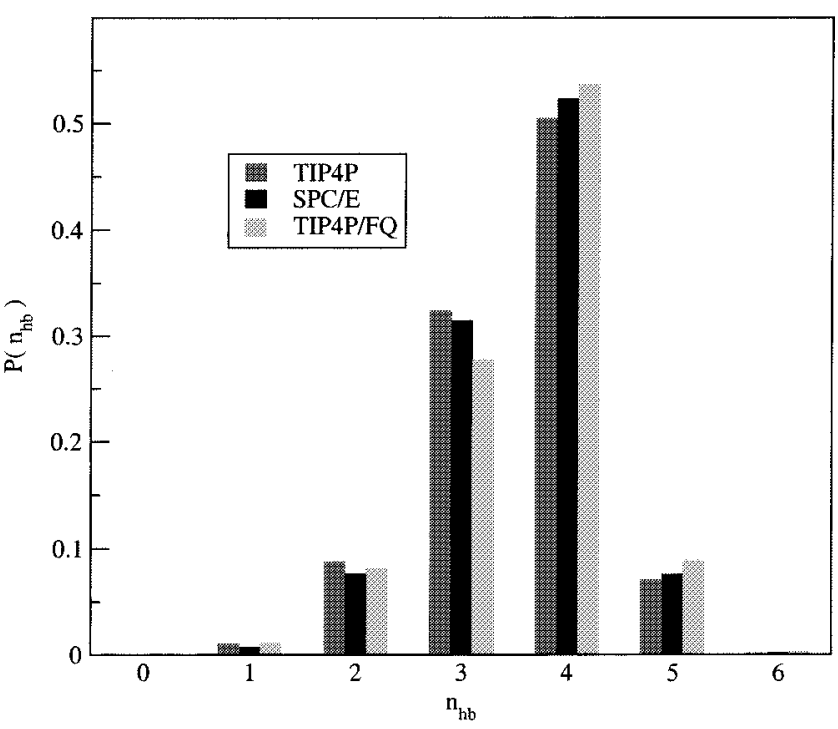

Figure 1. The distribution of the number of hydrogen bonds, as defined by the geometric criterion, a water molecule forms in liquid water. Only a slight difference exists between the polarizable and nonpolarizable models. The polarizable water model shows a stronger propensity to form hydrogen bonds.

rules were used for the Lennard-Jones interaction $\left(\sigma_{a b}=\right.$ $\sqrt{\sigma_{a} \sigma_{b}}, \epsilon_{a b}=\sqrt{\epsilon_{a} \epsilon_{b}}$ ) between the solute atoms and the water oxygen. All molecular dynamics runs were performed with 512 water molecules, and in the ethane and $\mathrm{NaCl}$ solutions, five solute molecules or pairs of solute counterions for an approximate solute concentration of $0.5 \mathrm{M}$. For each system, data were taken from five 100-ps trajectories run in the $N V E$ ensemble with the velocity Verlet integrator. Every $20 \mathrm{fs}$, a configuration is used in the following analysis. Each trajectory was started from a configuration sampled from a wellequilibrated NPT simulation using Nosé-Hoover chain (NHC) thermostats ${ }^{26}$ and an Andersen-Hoover-type barostat. ${ }^{27}$ All runs used cubic periodic boundary conditions and Ewald summation for the electrostatics. ${ }^{28}$ In terms of the box length $L(\approx 25 \AA)$, the real-space cutoff for the Ewald sum was $0.35 L$, the reciprocal-space cutoff was $7 \times 2 \pi / L$, and the screening parameter was set to $7.9 / \mathrm{L}$. The Lennard-Jones pair potential was truncated at $L / 2$.

\section{Hydrogen Bond Autocorrelation}

Autocorrelation analysis is used to characterize the structural relaxation of hydrogen bonds. The autocorrelation function $c(t)^{9,29}$ is defined as

$$
c(t)=\frac{\langle h(0) h(t)\rangle}{\langle h\rangle}
$$

where $h(t)=1$ if a tagged water pair is hydrogen bonded at time $t$, and $h(t)=0$ otherwise. Water-water hydrogen bonds are identified by a geometric criterion: a water pair is considered to be hydrogen bonded if the oxygen-oxygen distance is no greater than $3.5 \AA$, and simultaneously, the bonded $\mathrm{O}-\mathrm{H} \cdots \mathrm{O}$ angle is no less than $150^{\circ} .11$ The liquid structures of TIP4P, SPC/E, and TIP4P/FQ water models are similar enough that the same hydrogen bond definition should be valid for all three models. By using this definition, the distributions of the number of hydrogen bonds formed by a water molecule are only slightly different for the three models (Figure 1). A TIP4P water molecule forms an average of 3.54 hydrogen bonds, a SPC/E water molecule forms an average of 3.59 hydrogen bonds,

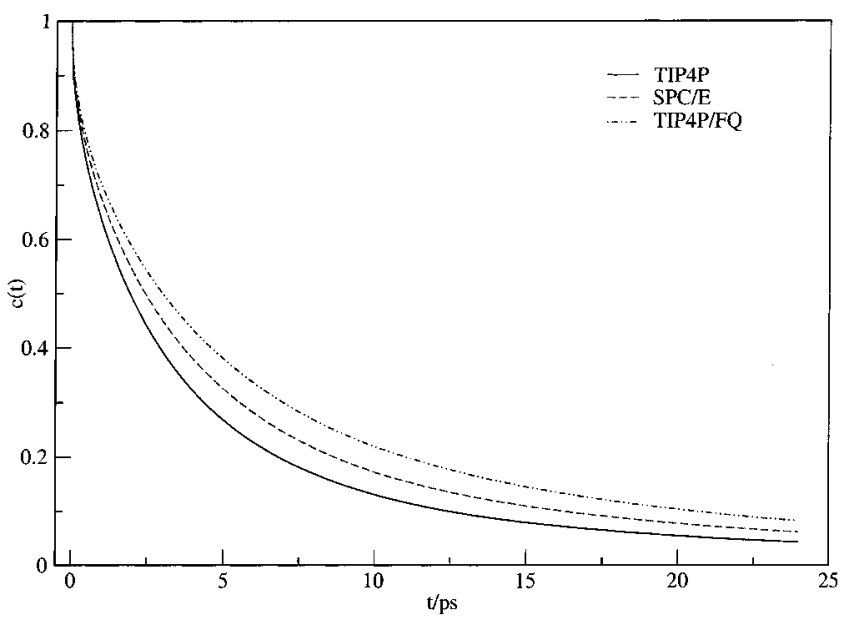

Figure 2. The hydrogen bond autocorrelation function $c(t)$ for TIP4P, SPC/E, and TIP4P/FQ water models. The hydrogen bonds relax much slower for the polarizable TIP4P/FQ model. The relaxation time $\tau_{\mathrm{rlx}}$, defined as $c\left(\tau_{\mathrm{rlx}}\right)=e^{-1}$, for the polarizable TIP4P/FQ model is 1.56 times longer than for the nonpolarizable TIP4P model, and 1.25 times longer than for the SPC/E model.

TABLE 1: Relaxation Time, $\tau_{\text {rlx }}$, and Lifetime, $\tau_{\mathrm{HB}}$, of Hydrogen Bonds in Neat Water for Different Water Models

\begin{tabular}{lccccc}
\hline water model & $\tau_{\mathrm{rlx}}$ & $\mathrm{k}$ & $\mathrm{k}^{\prime}$ & $\tau_{\mathrm{HB}}$ & $\tau_{\mathrm{rl} \times \mathrm{x}} \tau_{\mathrm{HB}}$ \\
\hline SPC/E & 4.20 & 0.35 & 0.78 & 2.86 & 1.47 \\
TIP4P & 3.32 & 0.45 & 1.02 & 2.22 & 1.49 \\
TIP4P/FQ & 5.26 & 0.29 & 0.67 & 3.44 & 1.53
\end{tabular}

The hydrogen bond lifetime is defined by eq 3 . The times are given in picoseconds. The unit for the rate constants $k$ and $k^{\prime}$ is picoseconds ${ }^{-1}$. $\tau_{\mathrm{rlx}}$ and $\tau_{\mathrm{HB}}$ are seen to be approximately related by $\tau_{\mathrm{rlx}} / \tau_{\mathrm{HB}}=1.5$, and therefore give qualitatively similarly descriptions of hydrogen bond kinetics.

whereas a TIP4P/FQ water molecule forms an average of 3.62 hydrogen bonds. TIP4P/FQ water thus shows a slightly stronger propensity for forming hydrogen bonds.

The statistical uncertainty of the calculated hydrogen bond autocorrelation functions was examined by comparing results obtained from averaging over independent subsets of simulation data. This uncertainty was much smaller than the variation in results obtained with different water models, indicating that the latter is statistically significant.

The polarizable model exhibits a much slower structural relaxation of hydrogen bonds than the nonpolarizable models (Figure 2). The relaxation times $\tau_{\mathrm{rlx}}$, defined as $c\left(\tau_{\mathrm{rlx}}\right)=e^{-1} c(0)$, are $\tau_{\mathrm{rlx}}{ }^{(\mathrm{TIP} 4 \mathrm{P} / \mathrm{FQ})}=5.26 \mathrm{ps}$ for the polarizable TIP4P/FQ model, $\tau_{\mathrm{rlx}}{ }^{(\mathrm{TIP} 4 \mathrm{P})}=3.32 \mathrm{ps}$ for the nonpolarizable TIP4P model, and $\tau_{\mathrm{rlx}}{ }^{(\mathrm{SPC} / \mathrm{E})}=4.20 \mathrm{ps}$ for the SPC/E model (Table 1). It is interesting to relate hydrogen bond kinetics with self-diffusion of liquid water. ${ }^{9}$ Hydrogen bonds hinder the self-diffusion of water, and faster hydrogen bond kinetics will result in faster diffusion. Conversely, broken hydrogen bonds can form again if the two water molecules have not diffused away from each other; therefore, slower diffusion will result in slower hydrogen bond relaxation. To eliminate the contribution of pair diffusion, we computed the following correlation function":

$$
n(t)=\frac{\langle h(0)[1-h(t)] H(t)\rangle}{\langle h\rangle}
$$

where $H(t)=1$ if the pair of water molecules are closer than $3.5 \AA$ at time $t$ and $H(t)=0$ otherwise. $n(t)$ is the time-dependent probability that the hydrogen bond is broken at time $t$ but the pair of water molecules have not diffused away. At long time, 


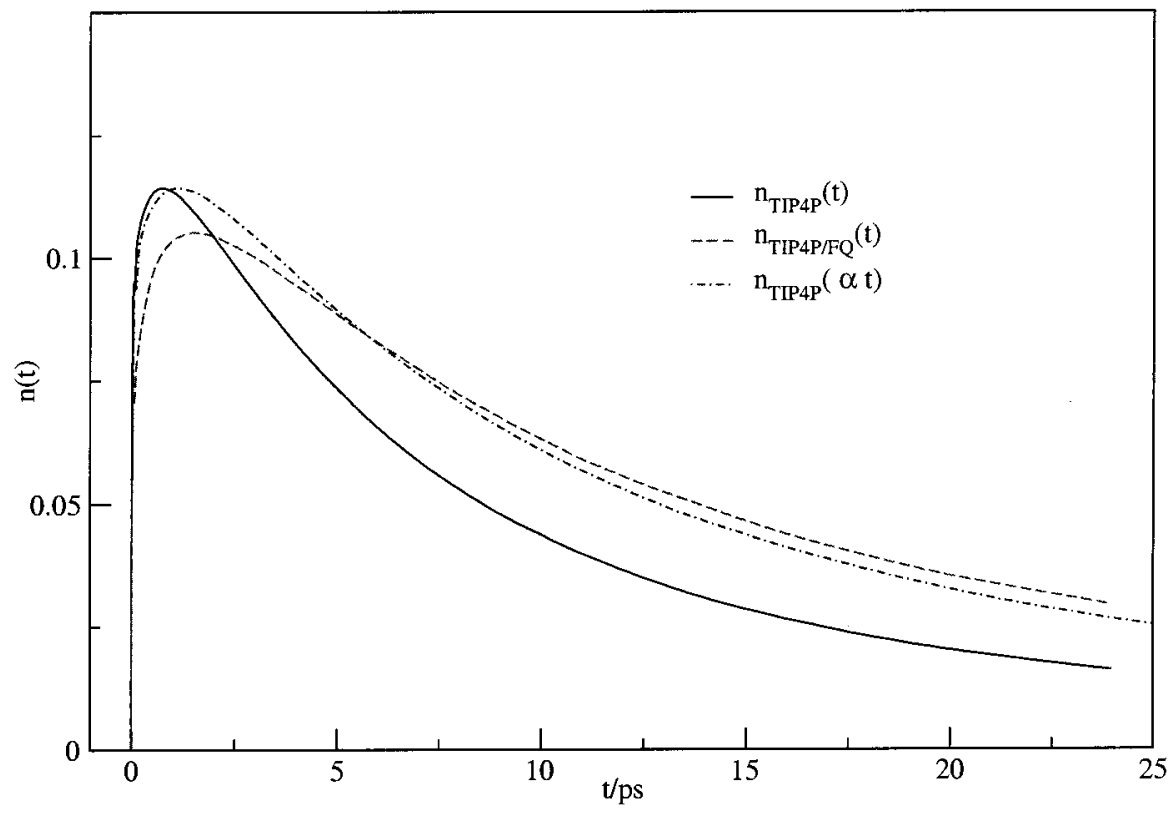

Figure 3. The time-dependent probability that a hydrogen bond is broken, but the water pair remains closer than $3.5 \AA$, $n(t)$, for the TIP4P and TIP4P/FQ models. Also shown is $n(t)$ for TIP4P model with time $t$ scaled by $\alpha=\tau_{\mathrm{R}}{ }^{\mathrm{TIP} 4 \mathrm{P} / \mathrm{FQ}} / \tau_{\mathrm{R}}{ }^{\mathrm{TIP}} 4 \mathrm{P}$, the ratio of the rotational time constant. The close agreement between $n_{\mathrm{TIP} 4 \mathrm{P}}(\alpha t)$ and $n_{\mathrm{TIP} 4 \mathrm{P} / \mathrm{FQ}}(t)$ supports the hypothesis that the long time behavior of $n(t)$ is determined by the rotational diffusion of water molecules.

the behavior of $n(t)$ is dominated by the rotational diffusion of water molecules. Therefore, the relaxation time scale for $n(t)$ is proportional to the rotational time constant $\tau_{\mathrm{R}}$, defined by the long-time behavior of the rotational correlation function. ${ }^{30} \tau_{\mathrm{R}}{ }^{\mathrm{TIP}} 4 \mathrm{P}$ $=1.4 \mathrm{ps}$ and $\tau_{\mathrm{R}}{ }^{\mathrm{TIP}} \mathrm{P} / \mathrm{FQ}=2.1 \mathrm{ps} .{ }^{17}$ Figure 3 shows the close agreement between $n_{\mathrm{TIP} 4 \mathrm{P}}(\alpha t)$ and $n_{\mathrm{TIP} 4 \mathrm{P} / \mathrm{FQ}}(t)$, where we scaled the time by $\alpha=\tau_{\mathrm{R}}{ }^{\mathrm{TIP}} 4 \mathrm{P} / \mathrm{FQ} / \tau_{\mathrm{R}}{ }^{\mathrm{TIP}} 4 \mathrm{P}$ for the $n(t)$ of the TIP4P model.

Luzar and Chandler proposed a simple model to describe hydrogen bond kinetics. ${ }^{9,31}$ In their model, they introduced the forward and backward rate constants, $k$ and $k^{\prime}$, for hydrogen bond breaking, and defined the hydrogen bond lifetime to be

$$
\tau_{\mathrm{HB}}=1 / k
$$

For long-time $t$, they related the reactive flux

$$
k(t)=-\frac{\mathrm{d} c(t)}{\mathrm{d} t}=-\frac{\langle\dot{h}(0)[1-h(t)]\rangle}{\langle h\rangle}
$$

with $c(t)$ and $n(t)$ by

$$
k(t)=k c(t)-k^{\prime} n(t)
$$

We used a least-squares fit for $t>1 \mathrm{ps}$ to produce the forward and backward rate constants $k$ and $k^{\prime}$ (Table 1) that best satisfy eq 5. To a good approximation, our relaxation times $\tau_{\mathrm{rlx}}$ are directly proportional to the hydrogen bond lifetimes $\tau_{\mathrm{HB}}$ defined in eq 3; therefore, they should give a qualitatively similar description of hydrogen bond kinetics as $\tau_{\mathrm{HB}}$.

\section{Cooperativity in Hydrogen Bond Kinetics}

To explore the cooperativity in hydrogen bond kinetics caused by water polarizability and concomitant many-body interactions, we calculated the autocorrelation function for hydrogen bonds in a different environment, distinguished by the number of hydrogen bonds each water of the concerned hydrogen bond forms. Specifically, we computed the following conditional correlation function ${ }^{11}$ :

$$
c_{m n}(t)=\frac{\left\langle p_{m n}(0) h(0) h(t)\right\rangle}{\left\langle p_{m n} h\right\rangle}
$$

where $p_{m n}(t)=1$ if at time $t$ one water molecule of the tagged pair forms $m$ hydrogen bonds, and the other forms $n$ hydrogen bonds; $p_{m n}(t)=0$ otherwise. $c_{m n}(t)$ describes the structural relaxation of the subset of hydrogen bonds whose water molecules are initially in the bonding states $(m, n) . c_{m n}(t)$ gives a different description of hydrogen bond kinetics than the conditional reactive flux $k_{m n}(t)=-\left\langle p_{m n}(0) \dot{h}(0)[1-h(t)]\right\rangle /\left\langle p_{m n} h\right\rangle$ introduced by Luzar and Chandler, ${ }^{11}$ because $-\mathrm{d} c_{m n}(t) / \mathrm{d} t$ differs from $k_{m n}(t)$ by $-\left\langle\dot{p}_{m n}(0) h(0)(1-h(t))\right\rangle /\left\langle p_{m n} h\right\rangle$ (see eq 8). For the nonpolarizable models, $c_{\mathrm{mn}}(t)$ shows little difference for typical initial bonding states $m=3,4 ; n=3,4$ (Figure 4), in agreement with the findings of Luzar and Chandler. ${ }^{11}$ The relaxation times for the hydrogen bonds in the typical initial bonding states are $\tau_{\mathrm{rlx}}{ }^{33}=3.22 \mathrm{ps}, \tau_{\mathrm{rlx}}{ }^{34}=3.36 \mathrm{ps}$, and $\tau_{\mathrm{rlx}}{ }^{44}=$ $3.54 \mathrm{ps}$. For the SPC/E model, the respective values are $\tau_{\mathrm{rlx}}{ }^{33}$ $=4.16 \mathrm{ps}, \tau_{\mathrm{rlx}}{ }^{34}=4.26 \mathrm{ps}$, and $\tau_{\mathrm{rlx}}{ }^{44}=4.43 \mathrm{ps}$. For the nonpolarizable models, $\tau_{\mathrm{rlx}}{ }^{33}, \tau_{\mathrm{rlx}}{ }^{34}$, and $\tau_{\mathrm{rlx}}{ }^{44}$ are only slightly different from each other. When water polarizability is included, however, $c_{\mathrm{mn}}(t)$ splits for different $(m, n)$ (Figure 5). The relaxation times for the hydrogen bonds are $\tau_{\mathrm{rlx}}{ }^{33}=4.71 \mathrm{ps}$, $\tau_{\mathrm{rlx}}{ }^{34}=5.18 \mathrm{ps}$, and $\tau_{\mathrm{rlx}}{ }^{44}=5.76 \mathrm{ps}$. The differences between the hydrogen bonds with different initial bonding states are significant. When a water molecule forms the ideal number of four hydrogen bonds, the near-tetrahedral structure in the vicinity induces the most favorable electronic configuration for strong hydrogen bonds, and the involved hydrogen bonds relax particularly slowly. A water molecule can occasionally form more than four hydrogen bonds by the geometric definition. Such a situation usually corresponds to the transition state where one hydrogen bond is being replaced by another. In the nonpolarizable models, $c_{45}(t)$ decays slightly faster than $c_{33}(t)$ (insets, Figure 4), suggesting that in the absence of polarizability, 

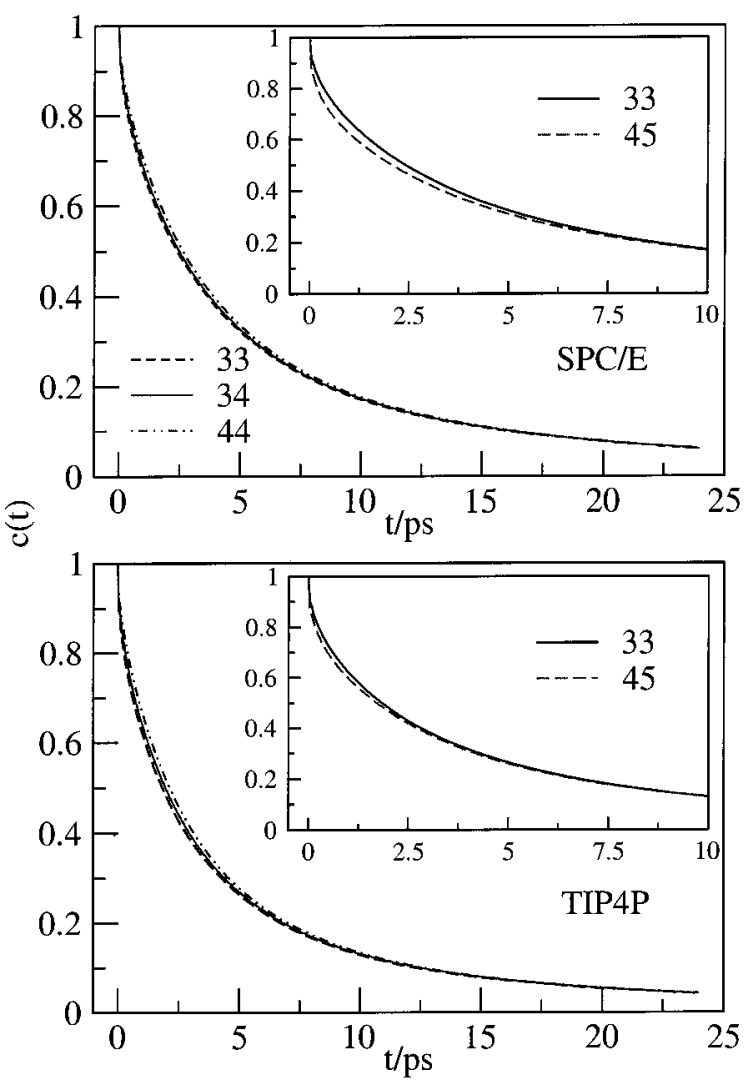

Figure 4. The conditional hydrogen bond autocorrelation functions for hydrogen bonds with different initial bonding states $(m, n)$ in nonpolarizable water models. For the nonpolarizable TIP4P and SPC/E model, hydrogen bond kinetics is uncorrelated with the bonding states of the involved water molecules. Despite the difference in the relaxation rates between the two models, the relaxation curves are qualitatively similar for the two models.

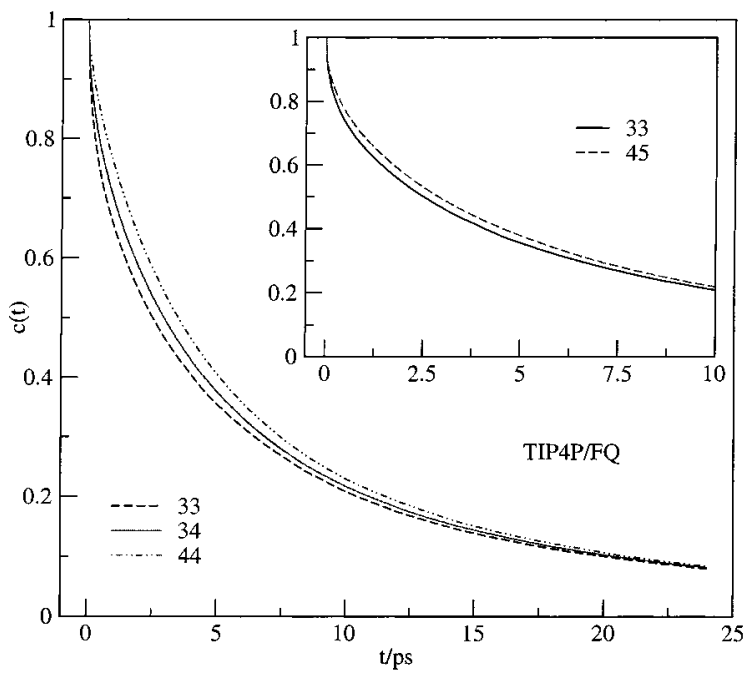

Figure 5. The conditional hydrogen bond autocorrelation functions for hydrogen bonds with different initial bonding states $(m, n)$ in the polarizable TIP4P/FQ water model. Hydrogen bond kinetics differ for different initial bonding states of the involved water molecules, suggesting cooperativity in the dynamic behavior of hydrogen bonds.

the tetrahedral structure of one water does not stabilize the hydrogen bond enough to compensate the disturbance of the intruding fifth water, which readily replaces the existing hydrogen bond. In the polarizable model, in contrast, $c_{45}(t)$ decays slower than $c_{33}(t)$ (inset, Figure 5). The tetrahedral structure induces a very favorable electronic configuration that stabilizes the hydrogen bonds, and it more than compensates the disturbance introduced by the fifth water. Thus, hydrogen bonds are apparently cooperative in their kinetic behavior when water polarizability is taken into account. Note that the difference in $c_{\mathrm{mn}}(t)$ vanishes at time $>10 \mathrm{ps}$, because the hydrogen bonds have sufficiently relaxed that the memory of the initial bonding states is lost.

\section{Hydrogen Bond Kinetics in the Solvation Shells of Various Solutes}

In another study, using a nonpolarizable water model, we found the kinetic behavior of water-water hydrogen bonds to differ significantly in the solvation shells of various residues of a polypeptide and its counterions. ${ }^{32}$ Near hydrophobic groups, the breaking of water-water hydrogen bonds is much slower than in bulk water. The slowdown is attributed to stronger hydrogen bonds and fewer water molecules to replace breaking hydrogen bonds near the hydrophobic groups. Around solvated cations such as $\mathrm{Na}^{+}$, hydrogen bonds break more rapidly than in bulk, because the strong electric field around the small $\mathrm{Na}^{+}$ distorts and weakens the nearby hydrogen bonds. Here we investigate the effect of water polarizability on hydrogen bond kinetics in solvation shells. Ethane is chosen as a representative hydrophobic molecule, and $\mathrm{NaCl}$ is used to represent ionic solutions.

The relaxation of hydrogen bonds is slower in ethane's solvation shell, for both the nonpolarizable and polarizable models (Figure 6). This agrees with our previous findings. Moreover, hydrogen bond kinetics near the hydrophobic group slows down to a greater degree for the polarizable water model than for the nonpolarizable model. The dimer energy between hydrogen-bonded water pairs is well defined for the nonpolarizable model. The distribution of such dimer energies for different environments shows that hydrogen bonds between two water molecules both in the solvation shell of ethane tend to be slightly stronger than hydrogen bonds in bulk (inset, Figure 6). We expect the same for the polarizable model, but the dimer energy is not well defined for polarizable models because of the many-body interactions. Polarizability causes the hydrogen bonds to mutually strengthen each other. Therefore the stronger hydrogen bonds in the solvation shell in turn strengthen the hydrogen bonds between the water in the solvation shell and the water in bulk. We speculate that for the polarizable model such indirect enhancement of hydrogen bond strength contributes partly to the slowdown in the kinetics of hydrogen bonds between bulk and solvation shell water.

The effect of polarizability increases with the electric field of the environment. In the $0.5 \mathrm{M}$ ionic $\mathrm{NaCl}$ solution, polarizability makes a significant difference in both ionic coordination numbers ${ }^{33}$ and hydrogen bond kinetics between polarizable and nonpolarizable models (insets, Figures 7 and 8). For the nonpolarizable TIP4P model, the ions have very little effect on the kinetics of the water-water hydrogen bonds, and the hydrogen bond autocorrelation functions are almost identical for neat water and $0.5 \mathrm{M} \mathrm{NaCl}$ solution. For the nonpolarizable SPC/E model, the structural relaxation of hydrogen bonds is slightly slower in the $\mathrm{NaCl}$ solution than in neat water, whereas for the polarizable TIP4P/FQ model, the slowdown is even greater. For the SPC/E model, Chandra has ascribed the slowdown to the presence of the ion atmosphere friction in addition to the water-water friction. ${ }^{10}$ The increased friction causes slower translational diffusion of water molecules in the ionic solution, which in turn preserves the hydrogen bonds longer. We also study the effect of the ions on the kinetics of 

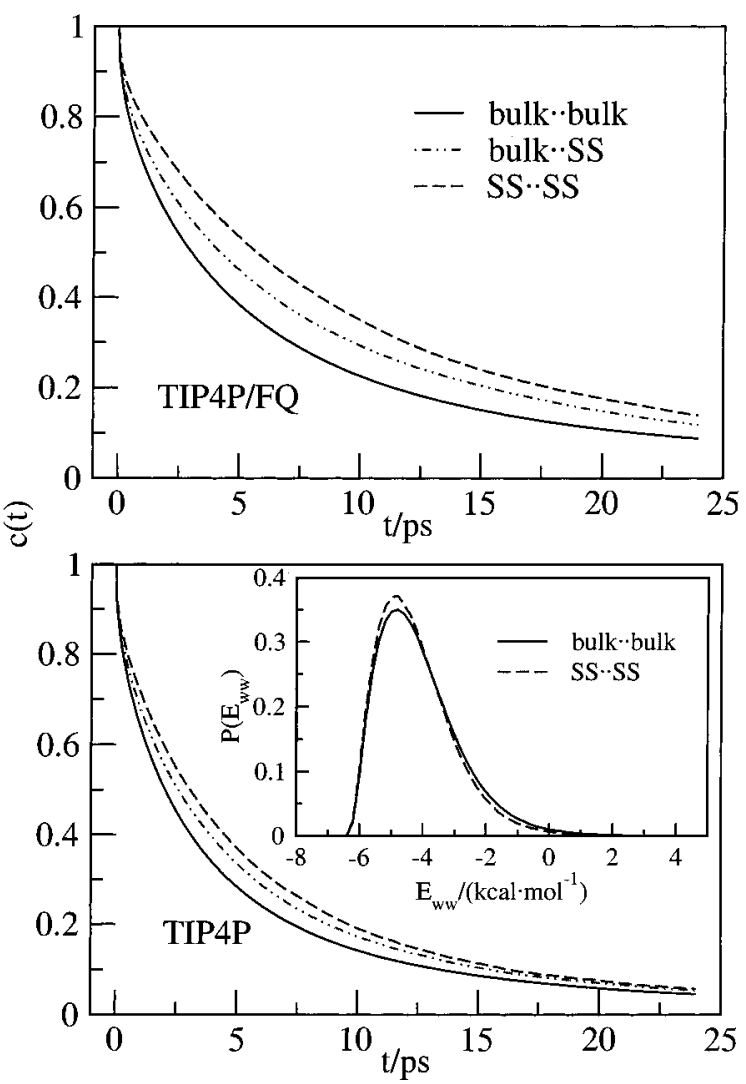

Figure 6. The autocorrelation function of hydrogen bonds in the solvation shell (SS) of ethane and in bulk water. Bulk • bulk represents the hydrogen bonds between two water molecules in bulk, bulk $\cdot S S$ represents the hydrogen bonds between a water molecule in bulk and another in the solvation shell of ethane, and SS*SS represents the hydrogen bonds between two water molecules both in the solvation shell of ethane. A water molecule is considered to be in the solvation shell of ethane if its oxygen atom is within $4.0 \AA$ from a carbon in ethane. ${ }^{34,35}$ The hydrogen bonds relax slower in the solvation shell of hydrophobic groups. The distribution of the dimer energy of hydrogenbonded water pairs in the solvation shell (inset, for TIP4P model) shifts slightly to the lower end. The average dimer energy of hydrogen bonded water pairs in the solvation shell is $E_{\mathrm{ww}}^{(\mathrm{SS})}=-4.12 \mathrm{kcal} \cdot \mathrm{mol}^{-1}$, slightly lower than the bulk value $\bar{E}_{\mathrm{ww}}{ }^{\text {(bulk) }}=-4.00 \mathrm{kcal} \cdot \mathrm{mol}^{-1}$. (Unfortunately, in the polarizable model, the many-body nature of the interaction prevents us from making a similar analysis.) A wider spread exists between $c(t)$ in different environments for the polarizable model than for the nonpolarizable model.

the hydrogen bonds between a water molecule in the first solvation shell of the ion and a water molecule in bulk. For the nonpolarizable TIP4P and SPC/E model, hydrogen bond kinetics is faster near the $\mathrm{Na}^{+}$ions than in neat water for time $<5 \mathrm{ps}$ (see Figure 7), because the water dipoles are aligned with the strong radial electric field arising from the small cations, thereby distorting the hydrogen bond geometries and weakening the hydrogen bonds. ${ }^{32}$ For the SPC/E model, hydrogen bond kinetics is slower near the $\mathrm{Cl}^{-}$ions than in neat water. We cannot explain the slowdown of hydrogen bond kinetics near the $\mathrm{Cl}^{-}$ions precisely, and hereby only offer a possible rationalization. Because the electric field is weaker around the $\mathrm{Cl}^{-}$ions than around the $\mathrm{Na}^{+}$ions, because of the larger size of the $\mathrm{Cl}^{-}$ions, it distorts the nearby hydrogen bonds to a lesser degree. The water molecules in the solvation shell of $\mathrm{Cl}^{-}$form weak hydrogen bonds with the $\mathrm{Cl}^{-}$, which restrict their mobility. The reduced mobility of the water molecules help to hold the hydrogen bonds longer. For the polarizable TIP4P/FQ model, the dipole induced by the ions strengthens the hydrogen bonds, which, together with the increased friction described above, leads
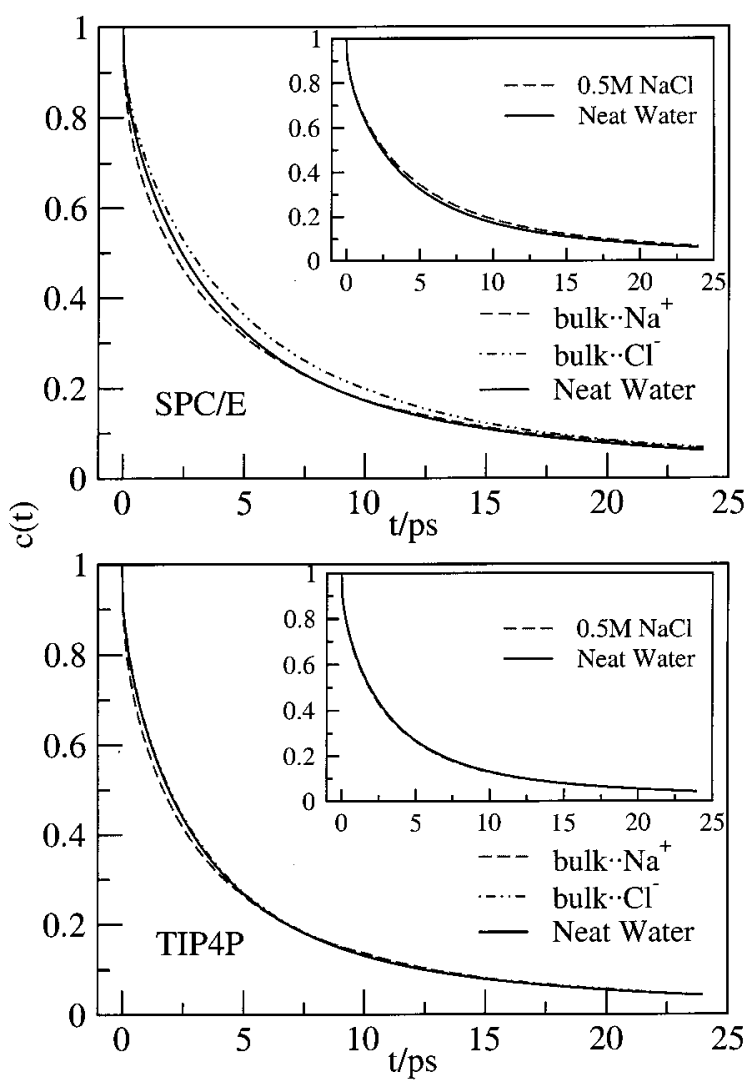

Figure 7. The autocorrelation functions for hydrogen bonds between a water molecule in bulk and another water molecule in the solvation shell of an ion, in $0.5 \mathrm{M} \mathrm{NaCl}$ solution of the nonpolarizable TIP4P and SPC/E models. (We consider a water molecule to be in the solvation shell of $\mathrm{Na}^{+}$if the oxygen- $\mathrm{Na}^{+}$distance is no greater than $3.25 \AA$, and of $\mathrm{Cl}^{-}$if the oxygen- $\mathrm{Cl}^{-}$distance is no greater than $3.80 \AA{ }^{33}$ ) The overall hydrogen bond autocorrelation functions in neat water and 0.5 $\mathrm{M} \mathrm{NaCl}$ solution are shown in the insets.

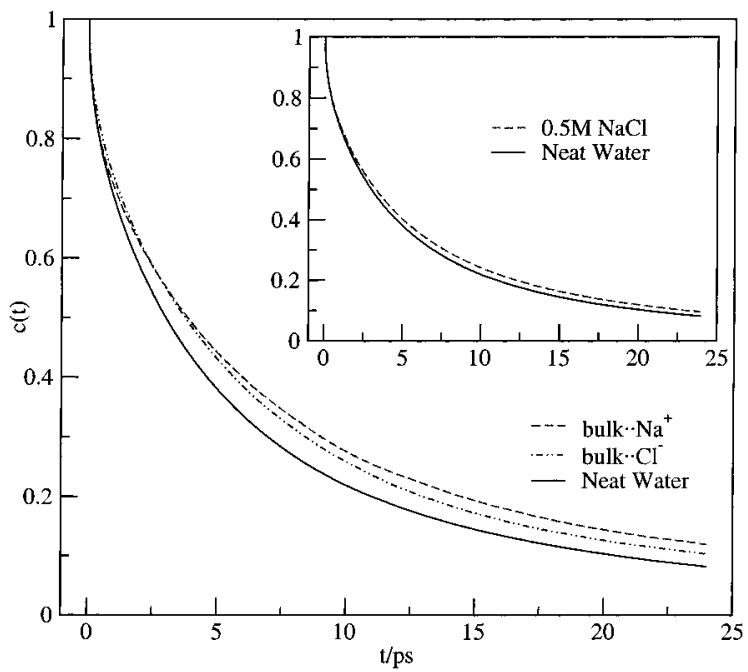

Figure 8. The autocorrelation functions for hydrogen bonds between a water molecule in bulk and another water molecule in the solvation shell of an ion, in $0.5 \mathrm{M} \mathrm{NaCl}$ solution of the polarizable TIP4P/FQ water model. The same definition of first hydration shell is used as for the nonpolarizable models. The overall hydrogen bond autocorrelation functions in neat water and $0.5 \mathrm{M} \mathrm{NaCl}$ solution are shown in the inset. $c(t)$ decays slower in $0.5 \mathrm{M} \mathrm{NaCl}$ than in neat water. The bulk $\cdot \mathrm{Na}^{+}$ hydrogen bonds relax slower than the bulk $\bullet$ bulk hydrogen bonds.

to an increase in their lifetime. Around the cations, the water dipoles point away from the cation, and the electrons are drawn toward the cation by the electric field. This results in an 
intramolecular charge transfer that increases the water dipoles. Around the anions, the dipoles point toward the anion, but the electrons are pushed away from the anion, resulting in a charge transfer that also increases the water dipoles. Therefore, polarizability always augments the water dipole and strengthens the hydrogen bonds around solvated ions. In contrast to the nonpolarizable water models, the bulk $\cdot \mathrm{Na}^{+}$hydrogen bonds relax more slowly for the polarizable TIP4P/FQ model than in neat water (Figure 8). The respective relaxation times are $\tau_{\mathrm{rlx}}{ }^{\text {neat }}$ $=5.26 \mathrm{ps}$ and $\tau_{\mathrm{rlx}}$ bulk $\cdot \mathrm{Na}^{+}=6.83 \mathrm{ps}$. Hydrogen bonds break more slowly near the $\mathrm{Na}^{+}$ions. The strong electric field around small $\mathrm{Na}^{+}$ions gives rise to a large induced dipole in surrounding water. This enhances the hydrogen bond strength near the ions, which more than offsets the distortion of the hydrogen bonds. Near the larger $\mathrm{Cl}^{-}$ions, the electric field is weaker, and consequently polarizability has a smaller, yet still pronounced effect. Hydrogen bond breaking slows down less around $\mathrm{Cl}^{-}$ions than around $\mathrm{Na}^{+}$(see Figure 8). As a result, for the polarizable model, hydrogen bond kinetics is slower around the $\mathrm{Na}^{+}$ions than around the $\mathrm{Cl}^{-}$ions, a reversal of the relationship compared with the nonpolarizable models.

\section{Transition State Theory Analysis}

To further quantify the effect of explicit polarizability, we compared the nonpolarizable TIP4P model and the polarizable TIP4P/FQ model, whose only difference is the polarizability in the TIP4P/FQ model, in the framework of the transition state theory. The transition state theory (TST) rate constant for breaking a hydrogen bond is given by

$$
k_{\mathrm{TST}}=-\left.\frac{\mathrm{d}}{\mathrm{d} t} c(t)\right|_{t \rightarrow 0^{+}}
$$

For the conditional autocorrelation function dependent on the environment $c_{m n}(t)$, we have

$$
\begin{aligned}
\frac{\mathrm{d}}{\mathrm{d} t} c_{m n}(t)=\left\langle p_{m n}(0) \dot{h}(0)[1-\right. & h(t)]\rangle /\left\langle p_{m n} h\right\rangle+ \\
& \left\langle\dot{p}_{m n}(0) h(0)[1-h(t)]\right\rangle /\left\langle p_{m n} h\right\rangle
\end{aligned}
$$

where we used the equilibrium condition $\left\langle p_{m n}(0) \dot{h}(0)\right\rangle+$ $\left\langle\dot{p}_{m n}(0) h(0)\right\rangle=\left.\left({ }^{d} / d t\right)\left\langle p_{m n}(t) h(t)\right\rangle\right|_{t=0}=0$. Because, on average, the first passage time of hydrogen bonds is about $0.3 \mathrm{ps}$, we have $\left\langle\dot{p}_{m n}(0) h(0)[1-h(t)]\right\rangle \approx 0$ for small $t$. Therefore, we can define the conditional transition state theory rate constant by

$$
k_{m n}{ }^{(\mathrm{TST})}=-\left.\frac{\mathrm{d}}{\mathrm{d} t} c_{m n}(t)\right|_{t \rightarrow 0+}=-\left.\frac{\left\langle p_{m n}(0) \dot{h}(0)[1-h(t)]\right\rangle}{\left\langle p_{m n} h\right\rangle}\right|_{t \rightarrow 0+}
$$

We assume that $k_{\text {TST }}$ obeys the Arrhenius relation:

$$
k_{\mathrm{TST}}=A e^{-\Delta G^{\ddagger} / R T}
$$

where $\Delta G^{\ddagger}$ is the activation Gibbs energy for breaking hydrogen bonds. The transition state rate constant can be quite sensitive to the choice of reaction coordinate. Thus, our inferred $\Delta G^{\ddagger}$ should not be regarded as quantitatively accurate but rather as a qualitative indication of the effects of polarizability on hydrogen bond kinetics. Prefactor $A$ depends on the transition state, which is identical for both models. We can therefore
TABLE 2: The Difference in the Relaxation Time of

\begin{tabular}{|c|c|c|c|c|c|c|}
\hline system & $\begin{array}{c}\text { environ- } \\
\text { ment }\end{array}$ & $\tau_{\mathrm{rlx}}{ }^{\mathrm{TIP}} 4 \mathrm{P}$ & $\tau_{\mathrm{rlx}}{ }^{\mathrm{TIP} 4 \mathrm{P} / \mathrm{FC}}$ & $\mathrm{Q}_{\mathrm{TST}}{ }^{\text {(TIP4P) }}$ & $\mathrm{k}_{\mathrm{TST}}{ }^{(\mathrm{TIP} 4 \mathrm{P} / \mathrm{FQ})}$ & $\Delta \Delta G^{\ddagger}$ \\
\hline \multirow{6}{*}{ neat water } & total & 3.32 & 5.26 & 2.56 & 2.00 & 0.146 \\
\hline & 33 & 3.22 & 4.71 & 2.51 & 2.43 & 0.019 \\
\hline & 34 & 3.36 & 5.18 & 2.31 & 1.92 & 0.111 \\
\hline & 35 & 2.86 & 4.22 & 3.95 & 3.54 & 0.066 \\
\hline & 44 & 3.54 & 5.76 & 2.00 & 1.32 & 0.248 \\
\hline & 45 & 3.11 & 5.22 & 3.42 & 2.24 & 0.252 \\
\hline \multirow{3}{*}{$\begin{array}{l}\mathrm{C}_{2} \mathrm{H}_{6} \\
\quad \text { solution }\end{array}$} & $\mathrm{B} \cdot \boldsymbol{B}$ & 3.53 & 5.34 & 2.52 & 2.01 & 0.133 \\
\hline & $\mathrm{B} \cdot \cdot \mathrm{SS}$ & 4.41 & 7.28 & 2.42 & 1.88 & 0.151 \\
\hline & SS $\cdot \cdot S S$ & 5.04 & 9.37 & 2.12 & 1.42 & 0.237 \\
\hline \multirow{3}{*}{$\begin{array}{l}\mathrm{NaCl} \\
\quad \text { solution }\end{array}$} & total & 3.24 & 5.77 & 2.64 & 1.98 & 0.171 \\
\hline & $\mathrm{B} \cdot \cdot \mathrm{SS}\left(\mathrm{Na}^{+}\right)$ & 3.11 & 6.83 & 3.10 & 2.12 & 0.226 \\
\hline & $\mathrm{B} \cdot \cdot \mathrm{SS}\left(\mathrm{Cl}^{+}\right)$ & 3.39 & 6.41 & 2.54 & 1.80 & 0.203 \\
\hline
\end{tabular}
Hydrogen Bonds $\tau_{\text {rlx }}$, the Transition State Theory Rate Constant $k_{\mathrm{TST}}$, and the Activation Gibbs Energy of Breaking Hydrogen Bonds between the Nonpolarizable and the Polarizable Models

"Total" stands for all the hydrogen bonds in the system. Bulk is abbreviated as B and solvation shell as SS. The unit for the relaxation time is picosecond, the unit for the rate constants $k_{\mathrm{TST}}{ }^{(\mathrm{TIP} 4 \mathrm{P})}$ and $k_{\mathrm{TST}}{ }^{(\mathrm{TIP} 4 \mathrm{P} / \mathrm{FQ})}$ is picosecond ${ }^{-1}$, and the unit for the difference in activation Gibbs energy $\Delta \Delta G^{\ddagger}$ is kilocalories per mole.

estimate the effect of polarizability on $\Delta G^{\ddagger}$ in a different environment. To that end, we computed

$$
\begin{aligned}
& \Delta \Delta G^{\ddagger}=\Delta G^{\ddagger(\mathrm{TIP} 4 \mathrm{P} / \mathrm{FQ})}-\Delta G^{\ddagger(\mathrm{TIP} 4 \mathrm{P})}= \\
& R T \ln \left(k_{\mathrm{TST}}{ }^{(\mathrm{TIP} 4 \mathrm{P})} / k_{\mathrm{TST}}{ }^{(\mathrm{TIP} 4 \mathrm{P} / \mathrm{FQ})}\right)
\end{aligned}
$$

As shown in Table 2, we see that polarizability invariably elevates the activation Gibbs energy for breaking hydrogen bonds. In neat water, $\Delta \Delta G^{\ddagger}$ generally increases with the number of hydrogen bonds the two water molecules form, and has a particularly large increase when a water molecule forms four hydrogen bonds of tetrahedral structure. This supports our speculation that, in the presence of polarizability, hydrogen bonds mutually strengthen each other. (We point out the exception when a water forms five hydrogen bonds. This usually corresponds to transition states where the hydrogen bonds are distorted and the mutual stabilization effect disappears.) In ethane solution, the increase in $\Delta G^{\ddagger}$ caused by polarizability is $0.1 \mathrm{kcal} \cdot \mathrm{mol}-{ }^{1}$ more for the hydrogen bonds between two water molecules both in the solvation shell than for those in bulk. Polarizability also increases $\Delta G^{\ddagger}$ for hydrogen bonds between bulk water and solvation shell water, partly because of the indirect enhancement of hydrogen bond strength as mentioned before. The elevation of $\Delta G^{\ddagger}$ is significant in $\mathrm{NaCl}$ solutions, because the polarization effect increases with the electric field because of the ions. The elevation of $\Delta G^{\ddagger}$ by polarizability in a different environment lies in the order:

tetrahedral bonding structure $>\mathrm{Na}^{+}>\mathrm{Cl}^{-}>$ other bonding structures (12)

where tetrahedral bonding structure corresponds to the situation when the water molecule forms four hydrogen bonds with other water molecules. Other bonding structures refer to situations in which the water molecule forms other than four hydrogen bonds.

Rapid recrossings occur in hydrogen bond kinetics, so that the TST rate constant, $k_{\mathrm{TST}}$, is considerably larger than the inverse relaxation time of the hydrogen bonds, $\tau_{\text {rlx }}$. This can be inferred from the time-dependent reactive flux of hydrogen bond breaking. (See, for example, refs 9 and 11.) 


\section{Conclusion}

We conclude that water polarizability causes hydrogen bonds to mutually strengthen each other. It significantly slows down hydrogen bond kinetics. It makes hydrogen bond kinetics cooperative and more dependent on the local environment. Thus water polarizability should not be ignored in hydrogen bond kinetics.

Acknowledgment. This material is based on work supported by National Science Foundation Grant CHE-00-76279.

\section{References and Notes}

(1) Eisenberg, D. S.; Kauzmann, W. The Structure and Properties of Water; Oxford University Press: New York, 1969

(2) Franks, F., Ed. Water, a Comprehensive Treatise; Plenum Press: New York, 1972-1982.

(3) Stillinger, F. H. Science 1980, 209, 451

(4) Chen, S.-H.; Teixeira, J. Adv. Chem. Phys. 1986, 64, 1, and references therein.

(5) Conde, O.; Teixeira, J. Mol. Phys. 1984, 53, 951 2150 .

6) Rousset, J. L.; Duval, E.; Boukenter, A. J. Chem. Phys. 1990, 92

(7) Sciortino, F.; Poole, P. H.; Stanley, H. E.; Havlin, S. Phys. Rev Lett. 1990, 64, 1686.

(8) Blumberg, R. L.; Stanley, H. E. J. Chem. Phys. 1984, 80, 5230

(9) Luzar, A.; Chandler, D. Nature 1996, 379, 55.

(10) Amalendu, C. Phys. Rev. Lett. 2000, 85, 768.

(11) Luzar, A.; Chandler, D. Phys. Rev. Lett. 1996, 76, 928.

(12) Luzar, A.; Chandler, D. J. Chem. Phys. 1993, 98, 8160.

(13) Starr, F. W.; Nielsen, J. K.; Stanley, H. E. Phys. Rev. E 2000, 62 579.

(14) Luzar, A. Faraday Discuss. 1996, 103, 29.

(15) Zhou, R.; Harder, E.; Xu, H.; Berne, B. J. J. Chem. Phys. 2001, 115,2348
(16) Murphy, F. W. J. Chem. Phys. 1977, 67, 5877.

(17) Rick, S. W.; Stuart, S. J.; Berne, B. J. J. Chem. Phys. 1994, 101, 6141

(18) Stuart, S. J.; Berne, B. J. J. Phys. Chem. 1996, 100, 11934

(19) Stuart, S. J.; Berne, B. J. J. Phys. Chem. A 1999, 103, 10300.

(20) Jorgensen, W. L.; Chandrasekhar, J.; Madura, J. D. J. Chem. Phys. $1983,9,926$.

(21) Berendsen, H. J. C.; Grigera, J. R.; Straatsma, T. P. J. Phys. Chem. 1987, 91, 6269.

(22) Car, R.; Parrinello, M. Phys. Rev. Lett. 1985, 55, 2471.

(23) van Belle, D.; Froeyen, M.; Wodak, S. J. Mol. Phys. 1992, 77, 239.

(24) Sprik, M. J. Phys. Chem. 1991, 95, 2283.

(25) Jorgensen, W. L.; Maxwell, D. S.; Tirado-Rives, J. J. Am. Chem. Soc. 1996, 118, 11225 .

(26) Martyna, G. J.; Klein, M. L.; Tuckerman, M. E. J. Chem. Phys. 1992, 97, 2635.

(27) Martyna, G. J.; Tobias, D. J.; Klein, M. L. J. Chem. Phys. 1994, 101,4177

(28) Allen, M. P.; Tildesley, D. J. Computer Simulation of Liquids; Oxford University Press: New York, 1987.

(29) Stillinger, F. H. Adv. Chem. Phys. 1975, 31, 1.

(30) Impey, R. W.; Madden, P. A.; McDonald, I. R. Mol. Phys. 1982 $46,513$.

(31) Luzar, A. J. Chem. Phys. 2000, 113, 10663.

(32) Xu, H.; Berne, B. J. J. Phys. Chem. B 2001, 105, 11929.

(33) The first minimum in the oxygen- $\mathrm{Na}^{+}$radial distribution function is about $3.25 \AA$. From the respective first minimum of each model, the corresponding coordination numbers are determined to be 6.1 for the TIP4P model, 5.8 for the SPC/E model, and 6.0 for the TIP4P/FQ model. The first minimum in the oxygen- $\mathrm{Cl}^{-}$radial distribution function is about 3.80 $\AA$. The corresponding coordination numbers are 7.3 for the TIP4P model, 7.2 for SPC/E model, and 6.4 for the TIP4P/FQ model.

(34) Cheng, Y.-K.; Rossky, P. J. Nature 1998, 392, 696.

(35) Mehrotra, P. K.; Beveridge, D. L. J. Am. Chem. Soc. 1980, 102, 4287. 